\title{
Roles for two RecA homologs in promoting meiotic chromosome synapsis
}

\author{
Beth Rockmill, ${ }^{1}$ Mary Sym, ${ }^{1,2}$ Harry Scherthan, ${ }^{3}$ and G. Shirleen Roeder ${ }^{1,4}$ \\ ${ }^{1}$ Department of Biology, Yale University, New Haven, Connecticut 06520-8103 USA; ${ }^{3}$ Department of Human Biology and \\ Human Genetics, University of Kaiserslautern, D-67653 Kaiserslautern, Germany
}

Previous studies have shown that the RAD51 and DMC1 genes of Saccharomyces cerevisiae encode homologs of the Escherichia coli RecA strand exchange enzyme. Results presented here demonstrate that the dmc1 and rad51 mutants undergo nearly complete chromosome synapsis, but synaptonemal complex formation is delayed substantially compared with wild type. In the zip1 mutant, chromosomes are paired homologously, but not synapsed, and the protein backbones (axial elements) of each pair of chromosomes are connected intimately to each other at a few sites referred to herein as axial associations. dmc1 zip1 and rad51 zip1 double mutants assemble axial elements that are not obviously associated, demonstrating that the Dmc1 and Rad51 proteins are required to establish or stabilize axial associations. We propose that axial associations serve to promote meiotic chromosome synapsis and that the absence of these associations accounts for the delayed and inefficient synapsis observed in $\mathbf{d m c 1}$ and rad51 strains. During meiosis in haploid yeast, chromosome synapsis takes place between nonhomologous chromosome segments. In a zip1 haploid, axial associations are not apparent, suggesting that these associations depend on interactions between homologous sequences.

[Key Words: Chromosome synapsis; synaptonemal complex; DMC1; MED1; RAD51; recombination; meiosis]

Received July 10, 1995; revised version accepted September 14, 1995.

The pairing of homologous chromosomes during meiotic prophase culminates in the formation of the synaptonemal complex (SC), which holds homologs closely apposed along their entire length (von Wettstein et al. 1984). Preparatory to SC formation, each chromosome begins to develop a dense, proteinaceous core called an axial element. In some organisms, axial elements become full length before synapsis initiates. In Saccharomyces cerevisiae, synapsis initiates at one or more sites on each homolog pair before the chromosomes have developed full-length axial cores (Padmore et al. 1991). At the sites of synaptic initiation, protein components of the central region of the SC assemble between the axial elements, which then become the lateral elements of the SC. Bidirectional SC extension results in full-length SCs. The central region of the complex consists of a central element, which lies between and parallel to the lateral elements, and of transverse filaments, which lie perpendicular to the long axis of the complex and span the space between lateral elements. Most of the chromatin is located outside the SC and is organized into loops that are attached to the lateral elements.

In some organisms the formation of SC has been shown to be preceded by an homology search that results

\footnotetext{
${ }^{2}$ Present address: Department of Biochemistry and Biophysics, University of California, San Francisco, California 94143-0554 USA.

${ }^{4}$ Corresponding author.
}

in the side-by-side alignment of homologous chromosomes at a distance greater than the width of the SC (von Wettstein et al. 1984). In yeast, this presynaptic alignment has been demonstrated by fluorescence in situ hybridization (FISH) using chromosome-specific DNA probes (Scherthan et al. 1992; Weiner and Kleckner 1994). Throughout this paper, the term pairing refers to the homologous alignment of chromosomes, both before and during $\mathrm{SC}$ formation, whereas synapsis refers specifically to SC assembly (Dresser and Giroux 1988; Alani et al. 1990; Sym et al. 1993).

The Zip1 protein of yeast localizes to synapsed chromosomes but not to unsynapsed axial elements, indicating that Zipl is a component of the central region of the SC (Sym et al. 1993). The predicted amino acid sequence of the Zip1 protein suggests that Zip1 forms an extended coiled coil (Cohen and Parry 1986) and the predicted length of a Zipl dimer corresponds approximately to the distance between lateral elements in the SC. Mutations that increase the length of the Zipl coiled coil lead to corresponding increases in the width of the SC, suggesting that Zipl is a component of transverse filaments (Sym and Roeder 1995). In spread meiotic chromosomes from the zip1 mutant, axial elements are lined up side by side in homologous pairs (Fig. 1D), but the distance between them is much greater and more variable than the distance between lateral elements in the SC (Fig. 1A) (Sym et al. 1993). At a few sites along each pair, the axial elements converge on each other and appear to be con- 

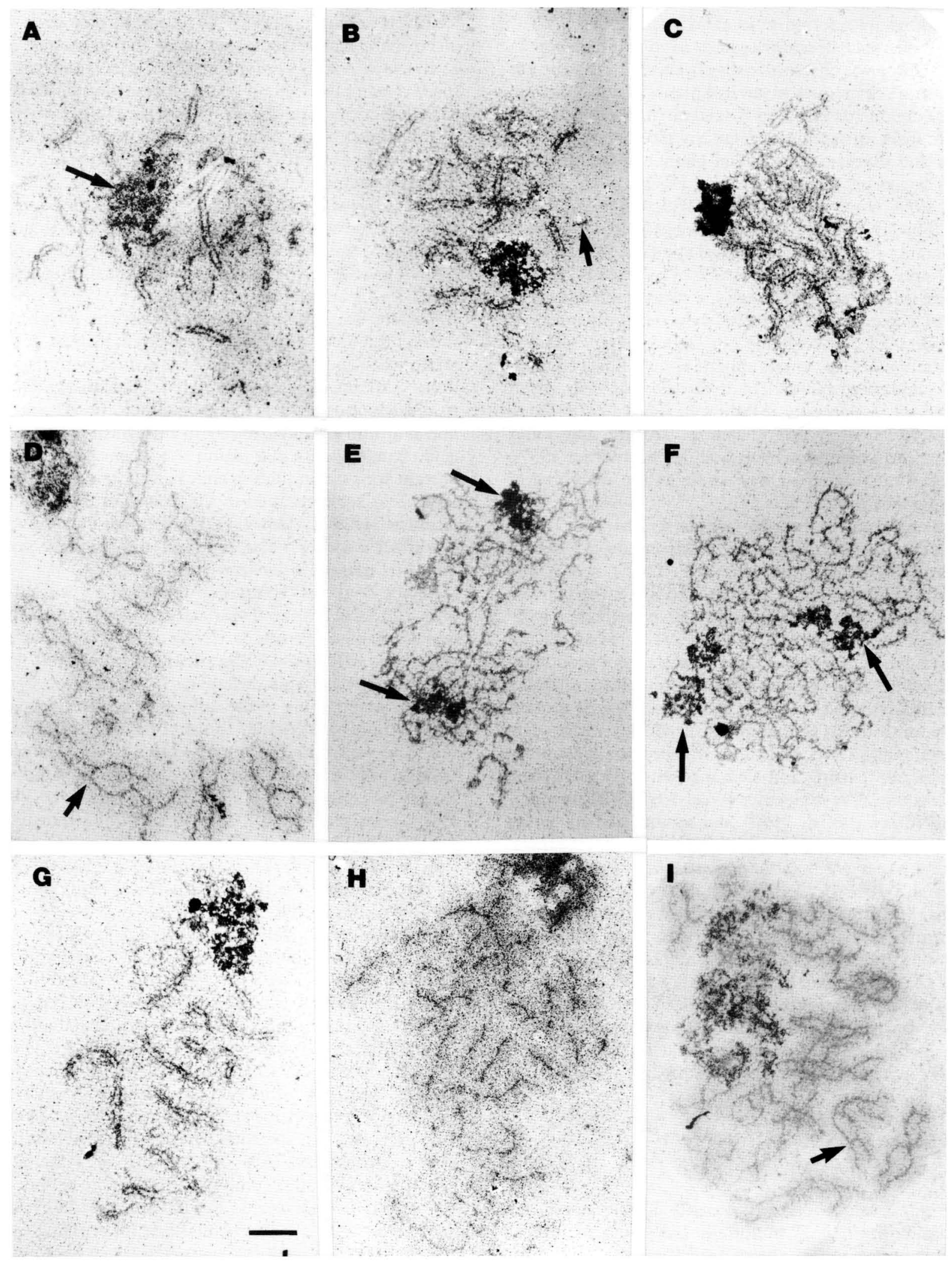

Figure 1. Electron micrographs of silver-stained spread chromosomes. $(A)$ Wild type (BR2495); $(B) d m c 1-3(\mathrm{BR} 2983) ;(C)$ dmc1::LEU2 (BR2960); (D) zip1 (MY63); (E) dmc1-3 zip1 (BR2984); (F) dmc1::LEU2 zip1 (BR2961); (G) rad51 (BR2962); (H) rad51 zip1 (BR2963); and (I) sep1 zip1 (BR2895). Spreads shown were prepared after $15(A, D), 16(G)$, or $20(B, C, E, F, H, I)$ hr of sporulation. The arrows in $A, E$, and $F$ point to nucleoli, the arrow in $B$ points to short axial elements, and the arrows in $D$ and $I$ point to axial associations. 
nected intimately (Fig. 1D). Throughout this paper these connections are referred to as axial associations.

The appearance of chromosomes in the zip1 mutant is similar to that of chromosomes observed during the zygotene stage of meiosis in several plants and in humans. In these organisms full-length axial elements develop before synapsis initiates, and regularly alternating convergences and divergences of axial elements are observed during zygotene (Rasmussen and Holm 1978; Hasenkampf 1984; Stack and Anderson 1986; Albini and Jones 1987). The sites of convergence between axial elements (presumably analogous to the axial associations observed in zip1 spreads) have been postulated to serve as sites of synaptic initiation; occasionally, a short piece of central element occupies the space between axial elements in the region of convergence. In appropriately stained spread preparations, small spherical or ellipsoidal nodules are observed frequently at the sites of association between axial elements (Albini and Jones 1987). These nodules, termed zygotene nodules (Albini and Jones 1987) or early recombination nodules (Carpenter 1987), have been postulated to play a role in the matching of homologous DNA sequences.

A recent study raises the possibility that the Dmcl and Rad51 proteins of yeast are components of early recombination nodules (Bishop 1994). Dmc1 and Rad51 colocalize to a number of discrete sites on chromosomes specifically during the zygotene stage of meiosis and the formation of Rad51-Dmc1 complexes depends on the action of gene products required for the initiation of meiotic recombination (Bishop 1994). Both Dmcl and Rad51 are homologs of the Escherichia coli RecA protein (Bishop et al. 1992; Shinohara et al. 1992), which coats single-stranded DNA to form a nucleoprotein filament and catalyzes pairing and exchange of strands between homologous DNA molecules (West 1992). Filament formation (Ogawa et al. 1993) and strand transfer (Sung 1994) have been demonstrated for Rad51, but not Dmc1.

In this paper we demonstrate that mutations in the $D M C 1$ and $R A D 51$ genes delay chromosome synapsis and that the Dmcl and Rad51 proteins are required for the formation or stabilization of axial associations. In addition, studies of meiosis in haploid yeast have revealed that axial associations are not established during synapsis between nonhomologous chromosomes. We propose that axial associations are sites of synaptic initiation, whose establishment depends on the recognition of DNA sequence homology by RecA-like proteins.

\section{Results}

med1-1 is an allele of DMCl

The med1-1 mutation was identified in a screen for sporulation-proficient, meiotic-lethal mutants (Rockmill and Roeder 1994). Characterization of this mutant revealed defects in meiotic recombination, homolog disjunction, and sister chromatid cohesion. By screening a yeast genomic library (Rose et al. 1987) for complementation of med1-1 spore lethality, a plasmid containing the MED1 gene was recovered. The cloned DNA was localized to chromosome V by Southern blot analysis of electrophoretically separated yeast chromosomes /Chu et al. 1986). A 3.25-kb XbaI fragment subcloned from the med1-1-complementing plasmid was found to retain complementing activity. From limited DNA sequencing of this plasmid and comparison with sequences in data bases, it was determined that this fragment contains two meiosis-specific genes, DMC1 (Bishop et al. 1992) and ISC10 (Kobayashi et al. 1993). Through complementation tests and analysis of subclones of the XbaI fragment, the med1-1 mutation was shown to be an allele of the $D M C 1$ gene (see Materials and methods). Henceforth, med1-1 will be referred to as $d m c 1-3$.

To determine the molecular basis of the $d m c 1-3 \mathrm{mu}-$ tation, the DMC1 gene was isolated from a med1-1 strain and sequenced (see Materials and methods). The dmc1-3 mutation effects a single transition of a $\mathrm{G}$ to an $\mathrm{A}$ at nucleotide position 387 , changing the glycine residue at amino acid position 99 to an arginine. This amino acid change occurs in a region that is thought to be important for protein folding and that contains several residues that are conserved in the RecA family of proteins (Story et al. 1993). However, the dmc1-3 mutation does not affect a conserved amino acid, and it is not obvious why this mutation perturbs Dmcl function.

Bishop et al. (1992) reported that a dmc1 null mutant (dmc1::LEU2) fails to sporulate and displays a uniform arrest in meiotic prophase. However, the dmc1-3 mutant sporulates efficiently (Rockmill and Roeder 1994). To determine whether this difference in sporulation competence is attributable to the difference in $d m c 1$ alleles or yeast strain background, the $d m c 1-3$ and $d m c 1:: L E U 2$ alleles were introduced into the same strain background by extensive backcrossing (see Materials and methods). In strains congenic with BR2495 (Rockmill and Roeder 1990), both mutants (BR2988 and BR2995) sporulate at $\sim 50 \%$ of the wild-type level and both mutants produce $23 \%$ viable spores ( 44 tetrads dissected for each). Thus, by these criteria, the $d m c 1-3$ and $d m c 1:: L E U 2$ mutants are indistinguishable.

Previous studies have shown that the spore inviability of a $d m c 1-3$ diploid is rescued by a spo13 mutation (Rockmill and Roeder 1994). spo13 causes meiotic cells to undergo a single round of chromosome segregation in which chromosomes can segregate either reductionally or equationally (Klapholz and Esposito 1980; Hugerat and Simchen 1993). Consequently, the spo13 mutation restores spore viability to many mutants defective in crossing-over and reductional division. The spo13 mutation increases the spore viability of the $d m c 1$ null mutant from $23 \%$ to $57 \%$ (BR2960); a congenic DMC1 spo13 strain (BR3021) produces $71 \%$ viable spores. Two hundred two-spore viable dyads from the $d m c 1:: L E U 2$ spo13 strain (BR2960) were analyzed for crossing-over in the HIS4-MAT interval. The map distance obtained was $14.9 \mathrm{cM}$, which is $39 \%$ of the corresponding wild-type level (Rockmill and Roeder 1990). When related strains were examined for crossing-over in the same interval, the map distance in a dmc1-3 diploid was $35 \%$ of wild 

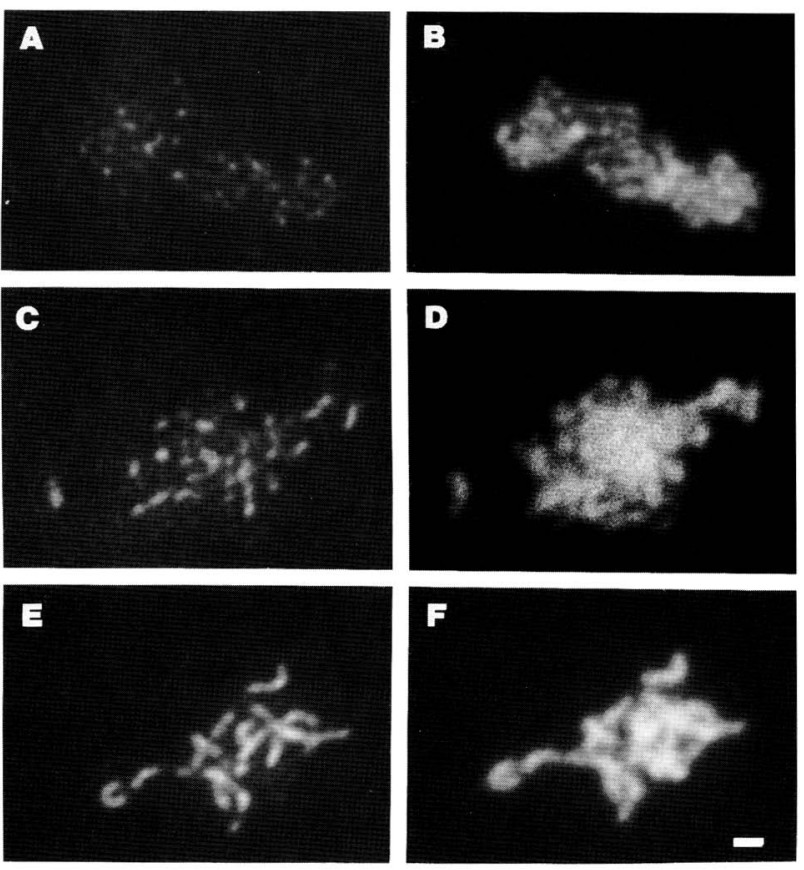

Figure 2. Categories of Zipl staining on spread meiotic nuclei. Spreads from the dmc1::LEU2 mutant (BR2960) $(A-D)$ or wild type (BR2495) $(E, F)$ were stained with anti-Zipl antibodies $(A, C, E)$ and a DNA-binding dye $(B, D, F)$. In the spreads shown in $A$ and $C, Z i p l$ staining is incomplete. The spread shown $(E)$ is a typical pachytene nucleus with full-length SCs.

type (Rockmill and Roeder 1994; see Materials and methods). Thus, these observations provide additional evidence that $d m c 1-3$ is a null allele.

\section{Chromosome synapsis is delayed in $\mathrm{dmcl}$ mutants}

To examine the kinetics of meiosis in $d m c 1$ strains, chromosome synapsis and nuclear division were monitored at various time points after the introduction into sporulation medium. Meiotic nuclei from wild type and mutant were surface spread and stained with anti-Zip1 antibodies to assay synapsis and with anti-tubulin antibodies to detect meiosis I and II spindles. Nuclei were classified as pachytene if Zipl was localized along the entire length of each chromosome pair (e.g., Fig. 2E). Nuclei were considered to be at intermediate stages in chromosome synapsis if they displayed incomplete staining with anti-Zipl antibodies (e.g., Fig. 2A,C).

After $12 \mathrm{hr}$ of sporulation, $\sim 40 \%$ of wild-type cells are in the pachytene stage of meiosis, whereas a similar number of cells are at intermediate stages in synapsis (Fig. 3A). After $12 \mathrm{hr}$, nuclei that stain with anti-Zipl antibodies decrease in frequency, whereas nuclei with spindles increase. By $16 \mathrm{hr}$, almost half of the wild-type cells are undergoing nuclear division.

Compared with wild type, both $d m c 1-3$ and $d m c 1:: L E U 2$ strains display delays in chromosome synapsis and nuclear division (Fig. 3B,C). Nuclei displaying incomplete Zipl staining persist for several hours and pachytene nuclei are not observed until $\sim 18 \mathrm{hr}$ of sporulation. By $18 \mathrm{hr}$, only a very small fraction of $d m c 1$ cells have progressed to the meiosis I division. After $24 \mathrm{hr}$ of sporulation, $\sim 25 \%$ of the cells contain spindles.

Spread meiotic chromosomes from wild type and $d m c 1$ mutants were stained with silver nitrate and examined in the electron microscope. At the time of maximum synapsis as determined by Zipl localization, most $d m c 1$ nuclei display extensive SC formation (see Fig. $1 \mathrm{~B}, \mathrm{C}$ ) and the SCs observed are similar to wild type (see

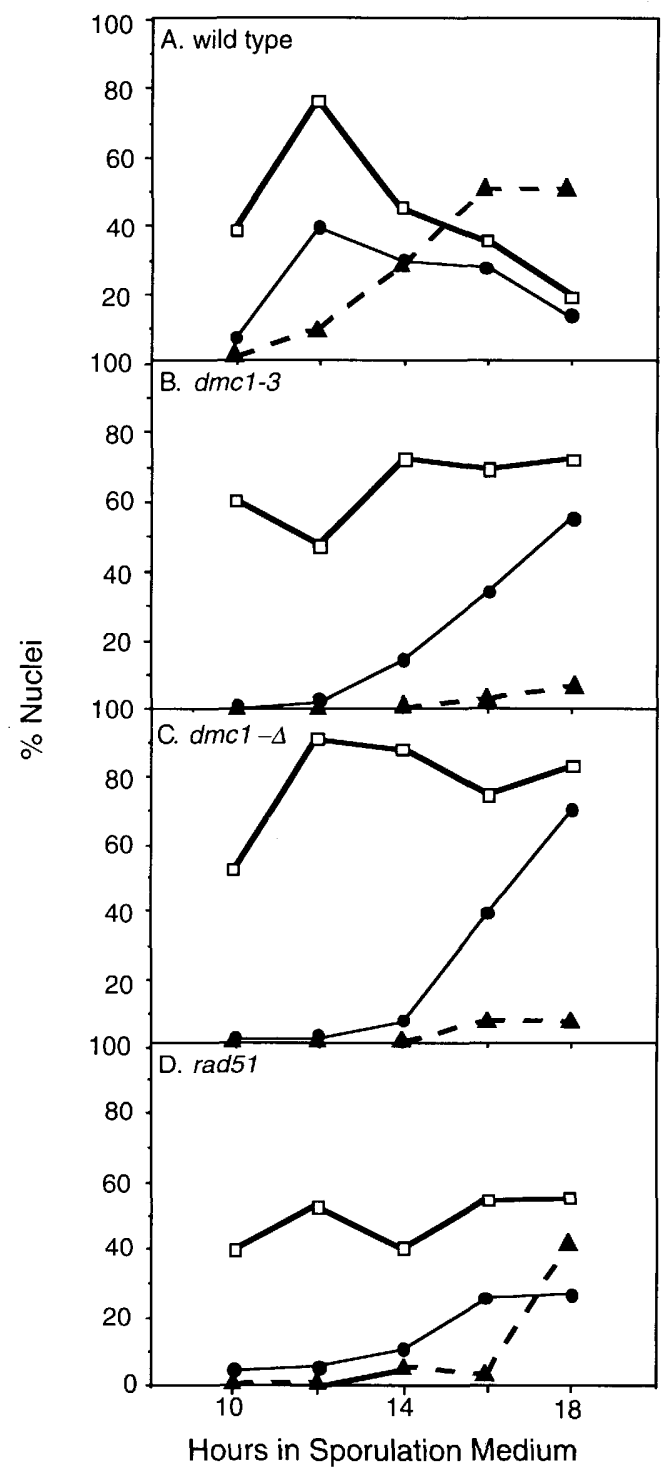

Figure 3. Time course of chromosome synapsis. Meiotic cells were spread and stained with anti-Zipl and anti-tubulin antibodies as described in Materials and methods. (ㅁ) All nuclei that stained with anti-Zipl antibodies, regardless of the extent of Zipl localization; $(O)$ only those nuclei in which most or all chromosomes had Zipl localized along their entire lengths, indicating complete synapsis; $(\mathbf{\Delta})$ nuclei with spindles. At least 100 spreads were scored at each time point. (A) Wild type (BR2495); (B) dmc1-3 (BR2983); (C) dmc1::LEU2 (BR2960); (D) rad51 (BR2962). 


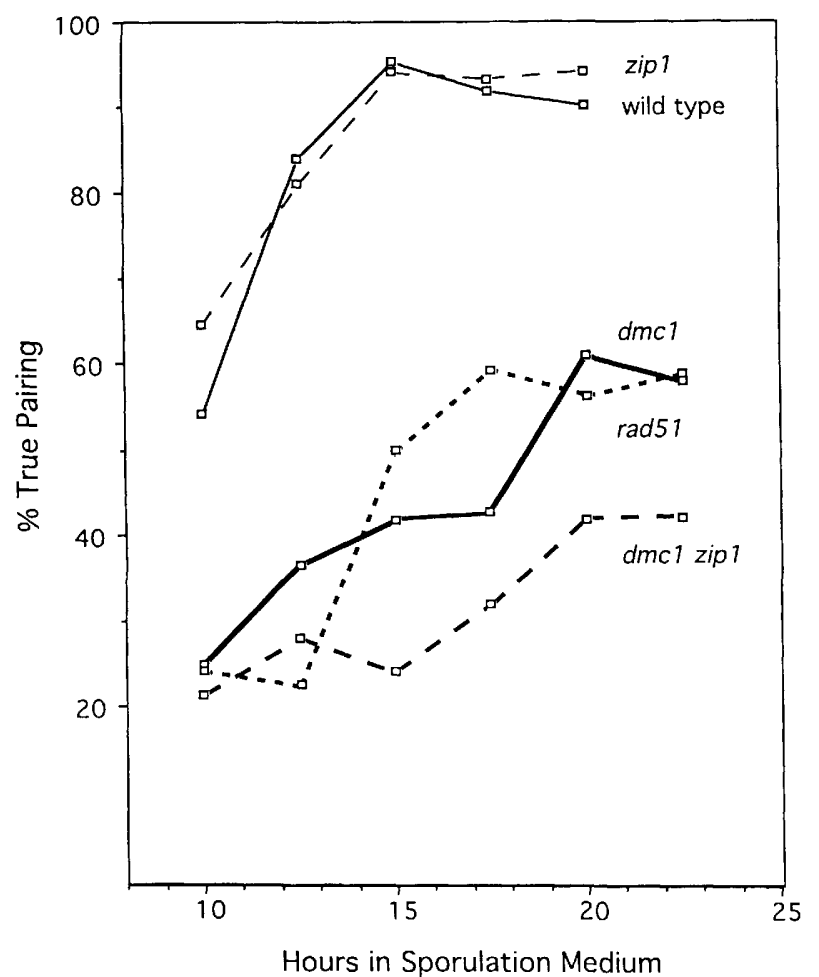

Figure 4. Time course of chromosome pairing. At various times of sporulation, the percentage of chromosomes I and III paired homologously was assayed by FISH. Strains assayed were BR2495 (wild type), BR2990 (zip1), BR2960 (dmc1::LEU2), BR2961 (dmc1::LEU2 zip1), and BR2962 (rad51). At least 100 nuclei with condensed FISH signals were scored for pairing at each time point. The percentage of nuclei with condensed chromosomes varied over the course of the experiment and reached maxima of $30 \%$ for wild type, $43 \%$ for zip $1,58 \%$ for dmc1::LEU2, 30\% for dmc1::LEU2 zip1, and $31 \%$ for rad51.

Fig. 1A) in morphology. In most spread nuclei, one or a few short unsynapsed axial elements are evident (e.g., Fig. 1B|, suggesting that small chromosomes frequently fail to synapse.

Two observations suggest that chromosome synapsis in $d m c 1$ strains involves homologous chromosomes in most or all cases. First, in silver-stained spread preparations, there is no evidence of axial elements folded back on themselves or of synapsis between axial elements of different length. Second, the ultimate extent of synapsis in $d m c 1$ cells is similar to that of wild type. Tracings of Zipl-stained chromosomes in pachytene nuclei revealed an overall SC contour length of $24 \mu \mathrm{m}$ for both strains $(24 \pm 2.8 \mu \mathrm{m}$ for wild type, $24 \pm 2.0 \mu \mathrm{m}$ for $d m c 1$, based on seven nuclei of each). Nonhomologous synapsis is generally incomplete, with part or all of some axial elements remaining unsynapsed (Loidl et al. 1991).

\section{Dmc1 is required to establish or stabilize axial associations}

To examine the role of the Dmc1 protein in the formation of axial associations, spread meiotic chromosomes from zip1 and dmc1 zip1 strains were stained with silver nitrate and examined in the electron microscope. In the zip1 mutant, axial elements of similar length are lined up side by side with one or more axial associations connecting each homolog pair. Axial associations are difficult to identify unambiguously; however, our counts indicate an average of $43( \pm 9$, based on 15 nuclei) associations per nucleus. The majority of axial associations are $\mathrm{H}$-shaped junctions, in which parallel axial elements do not contact each other directly but appear to be connected by a perpendicular bridge. Other axial associations are X-shaped junctions, in which the two axial elements appear to converge on each other, with no obvious intervening material. Some of the latter associations might be fortuitous overlaps resulting from spreading.

Nuclei from the $d m c 1$ zip1 double mutant look very different from zip1 nuclei, even at the time point of maximum synapsis in the $d m c 1$ single mutants. There is no obvious pairwise alignment of axial elements of similar length; rather, axial elements are arrayed in an apparently random manner. Furthermore, axial associations are either absent or substantially reduced in number (see Fig. 1E,F). Any connections observed are X-shaped junctions and, therefore, might not be bona fide axial associations. These observations suggest that the Dmcl protein is required to establish axial associations. Alternatively, dmc1 zip1 double mutants might form unstable axial associations that are unable to survive the spreading procedure.

\section{Chromosome pairing in the $\mathrm{dmc} 1$ zip1 double mutant}

Although axial associations are not evident in the $d m c 1$ zip1 double mutant, homologous chromosomes might still be paired homologously, albeit at a distance. To explore this possibility, FISH was carried out on spread meiotic nuclei (Scherthan et al. 1992). Chromosomes I and III were detected with red and green fluorochromes, respectively. Chromosomes were classified as unpaired if there were two separate signals of the same color in a single spread nucleus. Chromosomes were classified as

Table 1. Frequency of cells with two nucleoli

\begin{tabular}{|c|c|c|}
\hline Strain & $\begin{array}{l}\text { Relevant } \\
\text { genotype }\end{array}$ & $\begin{array}{l}\text { Percent of nuclei } \\
\text { with two nucleoli }\end{array}$ \\
\hline BR2495 & wild type & 4 \\
\hline BR2990 & zip1 & 4 \\
\hline BR2983 & $d m c 1-3$ & 5 \\
\hline BR2984 & $d m c 1-3$ zip1 & 24 \\
\hline BR2960 & $d m c 1-\Delta$ & 1 \\
\hline BR2961 & $d m c 1-\Delta$ zip 1 & 31 \\
\hline BR2962 & $\operatorname{rad} 51$ & 4 \\
\hline BR2963 & rad51 zip1 & 19 \\
\hline
\end{tabular}

Nucleoli were counted in silver-stained spreads of meiotic nuclei. Only nuclei containing SCs (for BR2495, BR2983, BR2960, and BR2962) or unsynapsed axial elements (for BR2990, BR2984, BR2961, and BR2963) were scored. Approximately 100 nuclei were scored for each strain. 
paired if their FISH signals touched each other or had fused into a single spot. Only nuclei containing clear and compact FISH signals were scored for homolog pairing; signal compaction depends on chromatin condensation, which reaches a maximum just before and during pachytene (Dresser and Giroux 1988; Kleckner et al. 1991; Loidl et al. 1994). The nuclei in which chromosome pairing can be assessed correspond to the nuclei in which axial elements, SC, or both, are observed in silverstained spread preparations.

Consistent with the kinetics of SC formation, homolog pairing in wild type is almost complete at $12.5 \mathrm{hr}$ and maximal at $15 \mathrm{hr}$, when $95 \%$ of the chromosomes are paired (Fig. 4). In the zip1 mutant, the kinetics and level of homolog pairing are indistinguishable from wild type. The $d m c 1$ null mutant displays a delay in chromosome pairing; the maximal level of pairing is reached by $20 \mathrm{hr}$ when nearly $60 \%$ of the chromosomes are paired. In the $d m c 1$ zip1 double mutant, maximal pairing occurs at $20 \mathrm{hr}$ when slightly $>40 \%$ of the chromosomes are paired. Thus, although the double mutant does undergo meiotically induced chromosome pairing, homolog alignment is reduced compared to the single mutants.

The behavior of the nucleolus also indicates a difference between single and double mutants. In wild-type diploids grown vegetatively, there is a single nucleolus, although the rRNA genes are located on both copies of chromosome XII (Granot and Snyder 1991). Similarly, in wild-type cells in meiotic prophase, there is a single nucleolus (Table 1), which stains intensely with silver nitrate (see Fig. 1A). Spread prophase nuclei from the $d m c 1$ and zip1 single mutants almost always contain a single nucleolus (Table 1 ). In contrast, $24 \%-31 \%$ of spreads from the $d m c 1$ zip1 double mutants contain two nucleoli. These observations suggest that in the absence of stable pairing of the chromosome XII homologs (i.e., in the absence of axial associations or $\mathrm{SC}$, the forces of meiotic chromatin condensation are sufficient to disrupt the associations between nucleolar organizing regions.

The $\mathrm{dmcl}$ mutation does not eliminate recombination in a zipl mutant

If axial associations are sites of processing of recombination intermediates, then a severe reduction in recombination in the dmc1 zip1 double mutant could account for the absence of axial associations. To assess recombi-

Table 2. Crossing-over in the HIS4-MAT interval

\begin{tabular}{llcc}
\hline Strain & $\begin{array}{l}\text { Relevant } \\
\text { genotype }\end{array}$ & $\begin{array}{c}\text { Map distance } \\
\text { (cM) }\end{array}$ & $\begin{array}{c}\text { Percent of } \\
\text { wild type }\end{array}$ \\
\hline BR2992 & wild type & 44.0 & 100 \\
BR2993 & zip1 & 61.3 & 139 \\
BR2974 & dmc1- & 13.6 & 31 \\
BR2996 & dmc1- zip1 & 13.6 & 31 \\
\hline
\end{tabular}

Crossing-over among meiotic prototrophs was measured as described in Materials and methods.

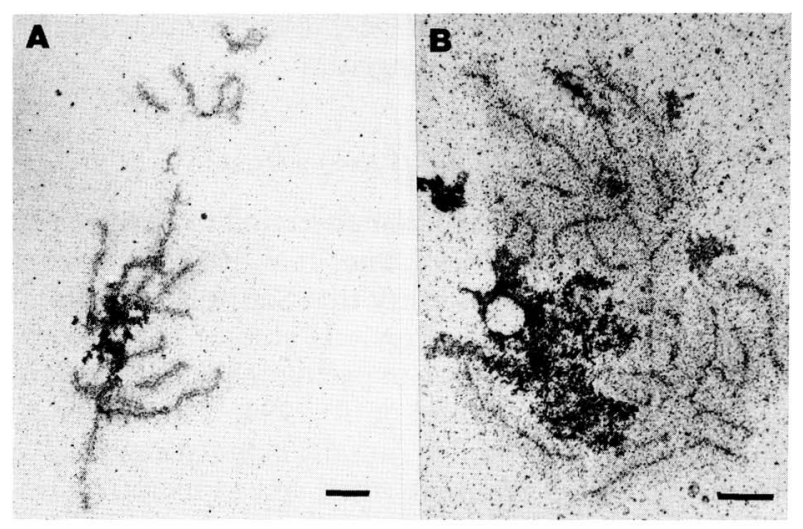

Figure 5. Electron micrographs of spread chromosomes from haploids. $(A)$ Wild type $(Y 20)_{i}(B)$ zip1 (MY248). Cells were spread after $30 \mathrm{hr}$ in sporulation medium.

nation in the double mutant, commitment to crossingover was measured among prototrophs resulting from meiotic gene conversion at the THR 1 locus on chromosome VIII. Thr ${ }^{+}$convertants were selected to enrich for cells that had undergone commitment to meiotic recombination. These prototrophs were then assayed for crossing-over in the HIS4-MAT interval on chromosome III (Table 2). Crossing-over in $d m c 1:: L E U 2$ diploids measured $31 \%$ of wild type, which is somewhat lower than the frequency observed among dissected dyads from a $d m c 1$ spo13 diploid. Compared to the zip1 single mutant, recombination in the $d m c 1$ zip1 double mutant is reduced 4.5 -fold. Thus, if axial associations are reduced to the same extent as crossovers, there should be 9 to 10 axial associations per nucleus in $d m c 1$ zip1 strains. Because no well-defined axial associations were observed in the double mutant, these data suggest that the effect of the $d m c 1$ mutation on the formation of axial associations is more severe than its effect on recombination.

\section{Rad51 is also required for stable axial associations}

Rad51 colocalizes with Dmcl and is necessary for Dmcl localization (Bishop 1994), predicting that Rad51 is also required to establish axial associations. The rad51 single mutant was monitored for chromosome synapsis, nuclear division, and homolog pairing. As observed in the $d \mathrm{mc1}$ mutants, nuclei with incomplete Zip1 staining persist for several hours and the formation of fully synapsed chromosomes is delayed substantially (see Fig. 3D). Homolog pairing is also slow and incomplete (Fig. 4).

Chromosomes from the rad51 single mutant and a rad51 zip1 double mutant were examined in the electron microscope. Whereas the rad51 single mutant makes normal SCs (see Fig. 1G), the double mutant makes axial elements that are not obviously paired and appear to lack the axial associations characteristic of zip1 strains (see Fig. $1 \mathrm{H}$ ). This analysis also revealed that $19 \%$ of nuclei from the double mutant contain two nucleoli (Table 1), 
indicating that rad51 (like $d m c 1$ ) disrupts nucleolar integrity in a zip1 mutant background.

\section{Sep1 is not required for axial associations}

The Sep1 protein has been demonstrated to have strand transfer activity in vitro (Kolodner et al. 1987; Dykstra et al. 1990), raising the possibility that Sepl plays a similar role to Rad51 and Dmc1. Previous studies have shown that the sep1 mutant arrests in pachytene with fully synapsed chromosomes (Bahler et al. 1994; Tishkoff et al. 1995). The sep1 zip1 double mutant is indistinguishable from the zip1 single mutant when spread meiotic chromosomes are examined in the electron microscope (see Fig. 1I). Thus, Sep1 is not required to establish axial associations.

\section{Axial associations are not formed during meiosis in a disomic haploid}

During meiosis in haploid yeast, there is extensive SC formation between nonhomologous chromosomes or chromosome segments (Loidl et al. 1991). If axial associations occur at sites of homology recognition or recombination intermediates, then such connections should be absent in haploids. To test this hypothesis, spread meiotic chromosomes from wild-type and zip1 haploids disomic for chromosome III were examined in the electron microscope. As expected, the wild-type strain undergoes extensive SC formation (Fig. 5A). The zip1 mutant makes axial elements that apparently lack axial associations between or within chromosomes (Fig. 5B). Axial associations between the two chromosome III homologs were not obvious; however, chromosome III is very small and therefore, is difficult to visualize in silverstained preparations.

\section{Discussion}

Synapsis is delayed and homolog pairing is reduced in $\mathrm{dmcl}$ and rad51 mutants

Our results demonstrate that chromosome synapsis is delayed substantially in the $d m c 1$ and rad51 mutants, but the ultimate extent of synapsis is approximately wild type. In a previous study, Bishop et al. (1992) did not observe any nuclei containing full-length SCs in a $d m c 1$ SK1 strain. However, subsequent analysis of $d m c 1$ SK1 cells harvested at relatively late time points in meiosis has revealed extensive Zipl localization (D. Bishop, pers. comm.), consistent with our studies of BR2495.

We also observed a significant reduction in homolog pairing in the $d m c 1$ and rad51 mutants, consistent with the results of Weiner and Kleckner (1994). In our analysis of $d m c 1$ and rad51 strains, the kinetics and level of homolog pairing as assessed by FISH closely paralleled the timing and extent of chromosome synapsis, as measured by staining with anti-Zipl antibodies. These results suggest that our FISH assay is relatively insensitive to the homolog alignment that normally precedes SC forma- tion (Scherthan et al. 1992; Weiner and Klecker 1994). Results obtained in a previous study also suggested that the early connections between homologs are especially fragile in a BR2495 strain background, and therefore, easily disrupted during spreading (Nag et al. 1995). At the time of maximum homolog pairing and $\mathrm{SC}$ formation in $d m c 1$ and rad51 strains, $\sim 60 \%$ of chromosomes I and III are paired homologously (see Fig. 4). The remainder could be unsynapsed or engaged in nonhomologous synapsis. We believe that they are unpaired because short unsynapsed axial elements were observed frequently in silver-stained spreads, and chromosomes I and III are two of the smallest yeast chromosomes. The level of homolog pairing is probably much higher for large chromosomes as suggested by the absence of long, unsynapsed axial elements in silver-stained preparations. In addition, dmc1 and rad51 nuclei almost always contain a single nucleolus, indicating that chromosome XII /the largest yeast chromosome) is paired.

\section{Dmc1, Rad51, and sequence homology are required to establish axial associations}

Our results demonstrate that the axial associations observed in spread meiotic chromosomes from the zip1 mutant are absent, or substantially reduced in number, in dmc1 zip1 and rad51 zip1 double mutants. These observations suggest that the Dmcl and Rad51 proteins are required to establish axial associations. Alternatively, some other proteins might establish connections, whereas Dmcl and Rad5l serve to stabilize them. Although axial associations have thus far been observed only in zip1 mutants, it is our operating assumption that such associations also form during meiosis in wild-type yeast. We further assume that Dmcl and Rad51 are required for the establishment or stabilization of axial associations in wild type, just as they are in zip1 strains.

Axial associations have not been observed in wild-type yeast, presumably because they are masked by SC. The establishment of axial associations might precede and be immediately followed by chromosome synapsis. Alternatively, axial associations might normally be established after synapsis, in the context of mature SC. In the latter case, Dmcl and Rad51 would promote the formation of a structure (e.g., a recombination intermediate) that is converted to an axial association after Dmcl and Rad51 have dissociated from the chromosomes. We favor the view that axial associations normally precede SC formation for the following reasons. First, a role for axial associations in promoting chromosome synapsis accounts economically for the delay in synapsis observed in the dmc1 and rad51 mutants. Second, the axial associations observed in zip1 strains are cytologically similar to those observed in other organisms (Rasmussen and Holm 1978; Hasenkampf 1984; Stack and Anderson 1986; Albini and Jones 1987), in which it is clear that the associations are established during zygotene. Third, the hypothesis that axial associations normally follow SC formation requires one to postulate the existence of two independent pathways, such that the morphogenesis of 
axial associations proceeds although SC formation is blocked.

Rad51 and Dmc1 colocalize to a number of discrete sites on chromosomes during the zygotene and early pachytene stages of meiosis (Bishop 1994). The average number of Dmcl- and Rad51-staining foci is $\sim 45$ at the time of maximum abundance (Bishop 1994), which is similar to the number of axial associations. As mentioned above, early recombination nodules have been observed at the sites of association between axial elements during the zygotene stage of meiosis in a number of organisms (Rasmussen ad Holm 1978; Stack and Anderson 1986; Albini and Jones 1987). These observations lead us to propose that axial associations are established at the sites of Dmcl and Rad51 localization. However, there is no evidence to exclude the possibility that axial associations and the sites of Dmcl/Rad51 localization do not coincide. In this latter scenario, it would have to be the case that the Dmc1 and Rad51 proteins promote interchromosomal interactions that are necessary prerequisites to the formation of axial associations.

During meiosis in haploids, SC is formed between nonhomologous chromosomal regions (Loidl et al. 1991). If axial associations are present at all sites of synaptic initiation, during both homologous and nonhomologous synapsis, then these connections should be observed in a zip1 haploid. However, axial associations were not observed in the haploid, suggesting a requirement for homology in establishing connections. The dependence on homology is consistent with the requirement for RecAlike proteins, which promote interactions between homologous DNA sequences. We cannot exclude the possibility of a low number of axial associations in haploids; however, if such exist, their number appears to be insufficient to account for the extensive synapsis observed. Axial associations might be established in haploids at sites of homology-dependent interactions between dispersed repeated sequences, such as transposable elements or tRNA genes.

\section{Are axial associations sites of SC initiation?}

Axial associations might promote chromosome synapsis by holding paired axial elements in sufficient proximity to permit a transverse filament to bind both elements simultaneously. In the absence of axial associations, the initiation of synapsis may depend on fortuitous collisions between axial elements. Once synapsis is initiated, bidirectional SC extension would lead to formation of a full-length complex. SC formation in $d m c 1$ and rad51 strains may be related mechanistically to the nonhomologous synapsis observed in haploids; both are independent of axial associations. However, in $d m c 1$ and rad51 strains, collisions between axial elements usually involve homologous chromosomes (as these are often paired), whereas encounters between axial elements in haploids are presumably random.

During meiosis in tetraploids, different regions of the same chromosome can synapse with different partners. Because pairing partner switches are generated by inde- pendently initiated stretches of SC, they can be used to estimate the number of initiation sites (Loidl 1986; Loidl and Jones 1986). On the basis of the analysis of tetraploid yeast, Loidl (1995) has concluded that there is an average of 22 sites of synaptic initiation per diploid genome, which is approximately half of the number of axial associations observed in zip1 strains. These results can be reconciled in several ways. First, axial associations might be potential sites of synaptic initiation, with only a subset being used in any given meiosis. Second, the number of axial associations formed in the zip1 mutant might be greater than in wild type. Synapsis in wild type might reach completion before all possible connections have formed. Third, the number of axial associations formed in the SK1 strain used to count pairing partner switches might be less than in the BR2495 strain used to count axial associations. Indeed, cytological studies indicate that axial associations in SK1 are either fewer in number or less well preserved during spreading (Sym et al. 1993; Sym and Roeder 1994). Finally, the number of synaptic initiation sites determined from pairing partner switches may be an underestimate of the true number, as it assumes that all synaptic initiation events are independent. However, formation of SC between one pair of homologs may bring those chromosomes into closer physical proximity and therefore predispose subsequent initiation events to involve the same homolog pair.

Although it is appealing to suppose that synapsis initiates at axial associations, we cannot rule out the possibility that SC formation initiates elsewhere, even in wild-type cells. In this case, the function of axial associations would be simply to establish a stable association between axial elements and thus facilitate indirectly the initiation of synapsis at distant locations.

\section{Axial associations may be sites of double-strand break processing}

A number of experiments have shed light on the relationship between SC formation and meiosis-specific double-strand breaks (DSBs), which serve to initiate most or all meiotic recombination events. DSBs are induced before synaptic initiation during meiosis in wildtype diploids (Padmore et al. 1991), and they are induced at the same level during meiosis in haploids (de Massy et al. 1994; Gilbertson and Stahl 1994). These observations exclude the possibility that initiation of synapsis between homologs is required for DSB formation. Disappearance of DSBs (as a result of conversion to later recombination intermediates) is concurrent with the initiation of synapsis (Padmore et al. 1991), and mutations that eliminate DSBs prevent chromosome synapsis (Alani et al. 1990; Loidl et al. 1994; Rockmill et al. 1995). These results are consistent with the proposal that early steps in the DSB repair pathway are required to initiate synapsis (Kleckner et al. 1991; Padmore et al. 1991) .

Other studies provide additional support for the notion that DSBs promote stable interactions between homologs (Goldway et al. 1993; Goldway and Simchen 1993). When fragments of chromosome III were assayed 
for the ability to promote meiosis I nondisjunction of chromosome III homologs, two regions with profound effects were found. At least one of these contains a hot spot for DSBs. Homolog nondisjunction was promoted by a plasmid that contains the DSB site, but lacks a centromere, indicating that missegregation was not a result of competition for spindle fiber attachment. In addition, crossing-over between the plasmid and one chromosome was associated with only a small subset of nondisjunction events. Thus, a reasonable interpretation of these results is that a plasmid containing a DSB site interferes with chromosome segregation by preventing pairing or synapsis between homologs. If synapsis normally initiates at a single site on chromosome III (Loidl 1995), then synapsis between the plasmid and one copy of chromosome III might well suffice to prevent synapsis between the intact chromosomes.

The number of axial associations and of Dmcl-and Rad51-staining foci is not sufficient to account for all meiotic recombination events. A wild-type diploid undergoes $\sim 90$ crossovers (Olson 1991) and nearly twice as many strand exchange reactions unaccompanied by reciprocal exchange (Fogel et al. 1983) for a total of $\sim 260$ events. The maximum number of sites of Dmcl localization is 62 (Bishop 1994). This number might be an underestimate if individual complexes are short lived and, therefore, only a subset is present on chromosomes at any given point in time. In zip1 cells arrested in meiotic prophase, Dmcl complexes are retained and the average number observed under these conditions is 40 (Bishop 1994) (i.e., similar to the number of axial associations|. Thus, although Rad51 and Dmcl may act at all sites of strand exchange, only a subset of these appear to give rise to stable associations between chromosomes.

\section{Dmc1: a eukaryotic RecA with a novel function?}

In previous studies the $d m c 1$ and rad51 mutants were characterized in the SK1 yeast strain background (Bishop et al. 1992; Shinohara et al. 1992). Physical assays suggested that these mutations confer similar defects in the processing of meiosis-specific DSBs. In both mutants, breaks occur at the wild-type level and are processed correctly to expose single-stranded tails; however, the breaks accumulate and the tails eventually become longer than their wild-type counterparts. Mature reciprocal recombinants are reduced 5- to 10 -fold. These results led to the proposal that the Rad51 and Dmcl proteins act at a specific step in DSB processing, presumably strand invasion (Bishop et al. 1992; Shinohara et al. 1992).

Our data suggest that Dmcl plays a less critical role in DSB repair than suggested previously. The $d m c 1$ mutant displays a reasonably high level of spore viability $(23 \%)$ in BR2495 and viability is improved almost to the wildtype level by a spo13 mutation. Because a failure to repair DSBs results in spore lethality even in spo13 strains (Petes et al. 1991), these data suggest that DSB repair can proceed in the absence of the Dmcl protein. To examine this possibility further, dyads were dissected from a $d m c 1$ spo13 haploid, in which a single unrepaired DSB should result in spore lethality. Spore viability in the $d m c 1$ spo13 haploid was indistinguishable from that of a $D M C 1$ spo13 haploid (74\%). Assuming that DSBs occur at the wild-type level in $d m c 1$ BR2495 strains, the observed reduction in interchromosomal recombination in $d m c 1$ diploids suggests that many breaks are repaired by exchange between sister chromatids, instead of nonsisters. Two observations support this notion. First, the dmc1 mutant shows an elevated level of intrachromosomal recombination (Bishop et al. 1992). Second, in another mutant (hop1) defective in chromosome synapsis (Hollingsworth and Byers 1989), almost all of the induced DSBs result in exchange between sister chromatids (Schwacha and Kleckner 1994).

Our data raise the possibility that the primary function of $\mathrm{Dmcl}$ is to establish axial associations, and thus to promote the initiation of SC formation. Therefore, Dmcl might be required, not for strand exchange per se, but rather to facilitate strand exchange specifically between nonsister chromatids. Rad51 and Dmcl might participate equally in the formation of axial associations or Rad51 might act more indirectly through its role in Dmcl localization. The DMC1 gene is expressed and functions specifically during meiosis (Bishop et al. 1992). We speculate that Dmcl interacts with other meiosisspecific gene products in a process that is specific to meiosis, namely chromosome synapsis.

\section{Materials and methods}

Yeast strains

Yeast strain genotypes are given in Table 3. Diploid strains isogenic with BR2495 (Rockmill and Roeder 1990) were derived either by transformation of the parental haploids BR1919-8B and BR1373-6D or by genetic crosses. To construct isogenic haploids by crosses, both MATa and MAT $\alpha$ versions of BR1919$8 \mathrm{~B}$ and BR1373-6D were generated by mating-type switching. Plasmid YEpHO containing the HO gene in YEpl3 (Jensen et al. 1983) was transformed into both haploids. The resulting diploids were sporulated and tetrads were dissected to recover isogenic haploids of botk mating types. Transformants of BR19198B MAT $\alpha$ were crossed to transformants of BR1919-8B MATa to generate double mutants. Similarly, transformants of BR13736D MATa and BR1373-6D MATa were crossed to each other.

Congenic derivatives of BR2495 were constructed by backcrosses starting with a $d m c 1$ null mutant in an SK1 background (strain 187; Bishop et al. 1992) and a dmc1-3 mutant in a strain closely related to BR2495 (Rockmill and Roeder 1994). Haploids carrying the mutant alleles were backcrossed eight times to both BR1919-8B and BR1373-6D or their isogenic derivatives. Haploids congenic with BR1919-8B were then mated to haploids congenic with BR1373-6D; the resulting diploids should be $99.6 \%$ identical to BR 2495 .

$\mathrm{His}^{+}$derivatives of his4-260/his4-280 diploids were selected on medium lacking histidine. To determine which his 4 allele had been converted, the diploids were sporulated and the alleles present in $\mathrm{His}^{-}$spores were determined in tests of mitotic recombination. BR2992, BR2993, BR2974, and BR2996 were derived from BR2625, BR2990, BR2960, and BR2961, respectively. Strain Y20 was described by Menees and Roeder (1989). 
Table 3. Yeast strains

\begin{tabular}{|c|c|}
\hline Strain & Genotype \\
\hline BR2495 & $\frac{\text { his4-280 leu2-27 MATa }}{\text { his4-260 leu2-3,112 MATa }} \frac{\text { arg4-8 }}{\text { ARG4 }} \frac{\operatorname{thr1-1}}{\text { thr1-4 }} \frac{\operatorname{trp} 1-1}{\operatorname{trp1-289}} \frac{\text { ura3-1 }}{\text { ura-3-1 }} \frac{\text { ade2-1 }}{\text { ade2-1 }} \frac{\text { cyh10 }}{\text { CYH10 }}$ \\
\hline BR2625 & isogenic to BR2495 but homozygous spo13::ura3-1 \\
\hline BR2895 & isogenic to BR2495 but homozygous sep1::URA3 zip1::LEU2 \\
\hline BR2960 & congenic to BR2495 but homozygous dmc1::LEU2 spo13::ura3-1 \\
\hline BR2961 & congenic to BR2495 but homozygous $d m c 1:: L E U 2$ zip $1::$ LEU2 spo13::ura3-1 \\
\hline BR2962 & isogenic to BR 2495 but homozygous rad51::URA3 \\
\hline BR2963 & isogenic to BR2495 but homozygous rad51::URA3 zip1::LEU2 \\
\hline BR2974 & isogenic to BR2495 but his4-260/HIS4 and homozygous dmc1::LEU2 spo13::ura3-1 \\
\hline BR2983 & congenic to BR2495 but homozygous dmc1-3 spo13::ura3-1 \\
\hline BR2984 & congenic to BR2495 but homozygous dmc1-3 zip1::LEU2 spo13::ura3-1 \\
\hline BR2988 & congenic to BR2495 but homozygous dmc1-3 \\
\hline BR2990 & congenic to BR2495 but homozygous zip1::LEU2 spo13::ura3-1 \\
\hline BR2992 & isogenic to BR2495 but his4-260/HIS4 and homozygous spo13::ura3-1 \\
\hline BR2993 & isogenic to BR2495 but his4-260/HIS4 and homozygous zip1::LEU2 spo13::ura3-1 \\
\hline BR2995 & congenic to BR 2495 but homozygous $d m c 1:: L E U 2$ \\
\hline BR2996 & congenic to BR2945 but his4-260/HIS4 and homozygous dmc1::LEU2 zip1::LEU2 \\
\hline BR3021 & congenic to BR2495 but homozygous spo13::ura3-1 \\
\hline Y20 & $\frac{\text { his4-260,39 leu2-112 }}{\text { his4-280 leu2-3 cry1 MATa ura3 trp1-H3 spo13::TRP1 ade2-1 lys2 cyh10 }}$ \\
\hline MY248 & isogenic to Y20 but zip1::LYS2 \\
\hline MY63 & isogenic to BR2495 but homozygous zip1::LEU2 \\
\hline 187 & MATa his4 dmc1::LEU2 ho::LYS2 lys2 ura3 \\
\hline
\end{tabular}

\section{Plasmids}

$\mathrm{pB} 156$, which is the original med1-1-complementing plasmid, contains $\sim 17 \mathrm{kbp}$ of yeast genomic DNA in YCp50 (Parent et al. 1985). Plasmid B174 contains a $3.25-\mathrm{kbp} \mathrm{XbaI}$ fragment of pB156 at the XbaI site of pRS316 (Sikorski and Hieter 1989). To construct $\mathrm{pB} 175, \mathrm{pB} 174$ was cut with $\mathrm{BamHI}$ and religated to remove the $5^{\prime}$ half of the $D M C 1$ coding region. $\mathrm{pB} 178$ was derived from $\mathrm{pB} 156$ by cutting with $\mathrm{XbaI}$ and religating; the resulting plasmid contains $\sim 6 \mathrm{~kb}$ of genomic sequences, but lacks the DMC1 and ISC10 coding regions. This plasmid was cut with $X b a I$ before transformation into yeast to clone the $X b a I$ fragment containing the DMC1 and ISC10 genes by gap repair (Rothstein 1991). This fragment was gap repaired from the original med1-1 mutant and a congenic wild type to generate $\mathrm{pB} 184$ and $\mathrm{pB} 183$, respectively.

Plasmids used to introduce the following gene disruptions were described previously: zip1::LEU2 (Sym et al. 1993), zip1::LYS2 (Sym and Roeder 1994), rad51::URA3 (Shinohara et al. 1992), dmc1::LEU2 (Bishop et al. 1992), and sep1::URA3 (Tishkoff et al. 1995). Petra Ross-Macdonald (Yale University, New Haven, CT) provided p6E, which carries the isc10::URA3 disruption. To create $\mathrm{p} 6 \mathrm{E}$, the $\mathrm{XbaI}-\mathrm{NdeI}$ fragment containing ISC10 was isolated from pNKY113 (Bishop et al. 1992) and inserted between the $\mathrm{XbaI}$ and BamHI sites of pHSS6 (Hoekstra et al. 1991). A 1.5-kb HpaI-KpnI fragment containing the URA3 gene from YEp352 (Hill et al. 1986) was then inserted between the $S s p I$ and $K p n I$ sites in the $I S C 10$ coding region. p6E was cut with NotI before transformation into yeast.

\section{Complementation tests}

To determine whether med1-1 is an allele of DMC1 or ISC10, the following experiments were carried out. In each case, complementation was assessed by measuring spore viability or meiotic gene conversion. First, $\mathrm{pB} 175$, which contains the entire ISC10 gene but only half of the DMC1 coding region, was used to transform a med1-1 homozygous diploid; the resulting trans- formants were similar to med1-1 in phenotype. Second, complementation tests were performed between a med1-1 haploid and $d m c 1$ and isc10 null mutants. The med1-1 mutant was complemented by isc10, but not by $d m c 1$. Third, the DMC1 and ISC10 genes were cloned from a med1-1 strain by gap repair; the resulting plasmid ( $\mathrm{pB} 184)$ did not complement the $d m c 1$ null mutant.

\section{Recombination assays}

Cells were grown vegetatively in YEPAD medium, which is YEPD (Rose et al. 1990) supplemented with adenine. Fresh overnight cultures were pelleted, resuspended in 7.5 volumes of $2 \%$ potassium acetate, and incubated with shaking at $30^{\circ} \mathrm{C}$. Crossing-over was measured among threonine prototrophs selected after $48 \mathrm{hr}$ of sporulation (Table 2). At least 450 threonine prototrophs from each strain were scored for histidine prototrophy and mating type. Recombinants in the HIS4-MAT interval were classified and map distance was calculated as described by Chua and Roeder (1995). The strains used for this analysis carry the spo13 mutation, so that ZIP1 cells that sporulate remain diploid and therefore, are comparable to the diploid products of unsporulated zip1 diploids.

Dissected dyads from spo13 strains were analyzed for crossing-over in the HIS4-MAT interval as described by Rockmill and Roeder (1990). The data presented by Rockmill and Roeder (1994) for med1-1 and congenic MED1 strains were reanalyzed as described by Rockmill and Roeder (1990) to permit comparison with the results obtained with the $d m c 1:: L E U 2$ mutant.

\section{Cytology}

For cytological analysis, cells were sporulated as described above for recombination assays. Spread meiotic nuclei were prepared as described by Dresser and Giroux (1988) except that glass slides were not precoated with plastic. Spreads to be examined in the electron microscope were stained with silver ni- 
trate (Howell and Black 1980), subsequently coated with plastic and lifted onto copper mesh grids (Loidl et al. 1991). Spreads for immunofluorescence were incubated overnight at $4^{\circ} \mathrm{C}$ with rabbit anti-Zipl antibodies (Sym et al. 1993) and rat anti-tubulin antibodies (Kilmartin et al. 1982) (YOL1/34, Sera-lab) diluted in PBS (150 mM NaCl, $50 \mathrm{mM} \mathrm{KPO}_{4}$ at $\mathrm{pH} 7.2$ ) plus $0.1 \%$ bovine serium albumin (BSA). The slides were washed first with PBS plus $0.1 \%$ BSA, then with PBS plus $0.1 \%$ BSA and $0.01 \%$ Nonidet P-40 and finally with PBS plus $0.1 \%$ BSA. Washed spreads were incubated with anti-rabbit IgG antibodies conjugated to Texas red (Jackson Labs) and anti-rat IgG antibodies conjugated to fluorescein isothiocyanate (Jackson Labs) for $2 \mathrm{hr}$ at room temperature. Spreads were then washed, stained with 4',6-diamidino-2-phenyl-indole, rinsed, and mounted in $1 \mathrm{mg} / \mathrm{ml}$ of p-phenylenediamine in $80 \%$ glycerol.

For FISH, spreads were prepared as for electron microscopy with modifications noted by Nag et al. (1995). FISH was carried out as described by Scherthan et al. (1992) and chromosome painting probes were generated and detected as described by Loidl et al. (1994). To calculate true pairing values, the frequency of associations between homologs was corrected for accidental pairings as indicated by the frequency of associations between nonhomologous chromosomes (Loidl et al. 1994). The corrected frequencies for chromosomes I and III were averaged.

\section{Sequencing}

Sequencing of the $d m c 1-3$ allele was carried out using primers based on the published DMC1 sequence (Bishop et al. 1992) using the Sequenase Kit (U.S. Biochemical) following the instructions of the supplier. The coding sequence derived from the wild-type $D M C 1$ gene in $\mathrm{pB} 183$ was found to be identical to the published sequence (Bishop et al. 1992).

\section{Acknowledgments}

We thank Josef Loidl, Douglas Bishop, Albert Smith, and Janet Novak for helpful discussions and comments on the manuscript. We are grateful to Petra Ross-Macdonald for providing the ISC10 gene disruption and to Douglas Bishop for providing SK1 strain 187. This work was supported by American Cancer Society grant VM-7C to G.S.R.

The publication costs of this article were defrayed in part by payment of page charges. This article must therefore be hereby marked "advertisement" in accordance with 18 USC section 1734 solely to indicate this fact.

\section{References}

Alani, E., R. Padmore, and N. Kleckner. 1990. Analysis of wildtype and rad50 mutants of yeast suggests an intimate relationship between meiotic chromosome synapsis and recombination. Cell 61: 419-436.

Albini, S.M. and G.H. Jones. 1987. Synaptonemal complex spreading in Allium cepa and A. fistulosum. I. The initiation and sequence of pairing. Chromosoma 95: 324-338.

Bahler, J., H. Hagens, G. Holzinger, H. Scherthan, and W.-D. Heyer. 1994. Saccharomyces cerevisiae cells lacking the homologous pairing protein $\mathrm{p} 175^{\mathrm{SEP} 1}$ arrest at pachytene during meiotic prophase. Chromosoma 103: 129-141.

Bishop, D.K. 1994. RecA homologues Dmcl and Rad51 interact to form discrete nuclear complexes prior to meiotic chromosome synapsis. Cell 79: 1081-1092.
Bishop, D., D. Park, L. Xu, and N. Kleckner. 1992. DMC1: A meiosis-specific yeast homolog of $E$. coli recA required for recombination, synaptonemal complex formation, and cell cycle progression. Cell 69: 439-456.

Carpenter, A.T.C. 1987. Gene conversion, recombination nodules, and the initiation of meiotic synapsis. BioEssays 6: 232-236.

Chu, G., D. Vollrath, and R.W. Davis. 1986. Separation of large DNA molecules by contour-clamped homogeneous electric fields. Science 234: 1582-1585.

Chua, P. and G.S. Roeder. 1995. Bdfl, a yeast chromosomal protein required for sporulation. Mol. Cell. Biol. 15: 36853696.

Cohen, C. and D.A. Parry. 1986. $\alpha$-Helical coiled coils: A widespread motif in proteins. Trends Biochem. Sci. 11: 245-248.

de Massy, B., F. Baudat, and A. Nicolas. 1994. Initiation of recombination in Saccharomyces cerevisiae haploid meiosis. Proc. Natl. Acad. Sci. 91: 11929-11933.

Dresser, M.E. and C.N. Giroux. 1988. Meiotic chromosome behavior in spread preparations of yeast. J. Cell Biol. 106: 567578.

Dykstra, C.C., R.K. Hamatake, and A. Sugino. 1990. DNA strand transfer protein $\beta$ (STP $\beta$ ) from yeast mitotic cells differs from strand transfer protein $\alpha$ from meiotic cells. J. Biol. Chem. 265: 10968-10973.

Fogel, S., R.K. Mortimer, and K. Lusnak. 1983. Meiotic gene conversion in yeast: Molecular and experimental perspectives. In Yeast genetics (ed. J.F.T. Spencer, D.M. Spencer, and A.R.W. Smith|, pp. 65-107. Springer-Verlag, New York.

Gilbertson, L.A. and F.W. Stahl. 1994. Initiation of meiotic recombination is independent of interhomologue interactions. Proc. Natl. Acad. Sci. 91: 11934-1 1937.

Goldway, M. and T.A.G. Simchen. 1993. Meiotic nondisjunction and recombination of chromosome III and homologous fragments in Saccharomyces cerevisiae. Genetics 133: 149158.

Goldway, M., A. Sherman, D. Zenvirth, T. Arbel, and G. Simchen. 1993. A short chromosomal region with major roles in yeast chromosome III meiotic disjunction, recombination and double strand breaks. Genetics 133: 159-169.

Granot, D. and M. Snyder. 1991. Segregation of the nucleolus during mitosis in budding and fission yeast. Cell Motil. Cytoskeleton 20: 47-54.

Hasenkampf, C.A. 1984. Synaptonemal complex formation in pollen mother cells of Tradescantia. Chromosoma 90: 275284.

Hill, J.E., A.M. Myers, T.J. Koerner, and A. Tzagoloff. 1986. Yeast/E. coli shuttle vectors with multiple unique restriction sites. Yeast 2: 163-167.

Hoekstra, M.F., H.S. Seifert, J. Nickoloff, and F. Heffron. 1991. Shuttle mutagenesis: Bacterial transposons for genetic manipulations in yeast. Methods Enzymol. 194: 329-342.

Hollingsworth, N.M. and B. Byers. 1989. HOP1: A yeast meiotic pairing gene. Genetics 121: 445-462.

Howell, W.M. and D.A. Black. 1980. Controlled silver-staining of nucleolus organizer regions with a protective collodial developer: A 1-step method. Experientia (Basel) 36: 1014 1015.

Hugerat, Y. and G. Simchen. 1993. Mixed segregation and recombination of chromosomes and YACs during single-divi. sion meiosis in spo13 strains of Saccharomyces cerevisiae. Genetics 135: 297-308.

Jensen, R., G.F. Sprague, and I. Herskowitz. 1983. Regulation of yeast mating-type interconversion: Feedback control of $H O$ gene expression by the mating-type locus. Proc. Natl. Acad. Sci. 80: 3035-3039. 
Kilmartin, J.V., B. Wright, and C. Milstein. 1982. Rat monoclonal antitubulin antibodies derived by using a new nonsecreting rat cell line. J. Cell Biol. 93: 576-582.

Klapholz, S. and R.E. Esposito. 1980. Recombination and chromosome segregation during the single division meiosis in spo12-1 and spo13-1 diploids. Genetics 96: 589-611.

Kleckner, N., R. Padmore, and D.K. Bishop. 1991. Meiotic chromosome metabolism: One view. Cold Spring Harbor Symp. Quant. Biol. 56: 729-743.

Kobayashi, M., Y. Hotta, and S. Tabata. 1993. Isolation and characterization of a yeast gene that is homologous with a meiosis-specific cDNA from a plant. Mol. \& Gen. Genet. 237: 225-232.

Kolodner, R., D.H. Evans, and P.T. Morrison. 1987. Purification and characterization of an activity from Saccharomyces cerevisiae that catalyzes homologous pairing and strand exchange. Proc. Natl. Acad. Sci. 84: 5560-5564.

Loidl, J. 1986. Synaptonemal complex spreading in Allium. II. Tetraploid A. vineale. Can. J. Gent. Cytol. 28: 754-761.

1995. Meiotic chromosome pairing in triploid and tetraploid Saccharomyces cerevisiae. Genetics 139: 15111520.

Loidl, J. and G. Jones. 1986. Synaptonemal complex spreading in Allium. I. Triploid A. sphaerocephalon. Chromosoma 93: $420-428$.

Loidl, J., K. Nairz, and F. Klein. 1991. Meiotic chromosome synapsis in a haploid yeast. Chromosoma 100: 221-228.

Loidl, J., F. Klein, and H. Scherthan. 1994. Homologous pairing is reduced but not abolished in asynaptic mutants of yeast. $J$. Cell Biol. 125: 1191-1200.

Menees, T.M. and G.S. Roeder. 1989. MEI4, a yeast gene required for meiotic recombination. Genetics 123: 675-682.

Nag, D.K., H. Scherthan, B. Rockmill, J. Bhargava, and G.S. Roeder. 1995. Heteroduplex DNA formation and homolog pairing in yeast meiotic mutants. Genetics 141: 75-86.

Ogawa, T., X. Yu, A. Shinohara, and E.H. Egelman. 1993. Similarity of the yeast RAD51 filament to the bacterial RecA filament. Science 259: 1896-1899.

Olson, M.V. 1991. Genome structure and organization in Saccharomyces cerevisiae. In The molecular and cellular biology of the yeast Saccharomyces cerevisiae: Genome dynamics, protein synthesis, and energetics (ed. J.R. Broach, J.R. Pringle, and E.W. Jones), pp. 1-39. Cold Spring Harbor Laboratory Press, Cold Spring Harbor, New York.

Padmore, R., L. Cao, and N. Kleckner. 1991. Temporal comparison of recombination and synaptonemal complex formation during meiosis in S. cerevisiae. Cell 66: 1239-1256.

Parent, S.A., C.M. Fenimore, and K.A. Bostian. 1985. Vector systems for the expression, analysis and cloning of DNA sequences in S. cerevisiae. Yeast 1: 83-138.

Petes, T.D., R.E. Malone, and L.S. Symington. 1991. Recombination in yeast. In The molecular and cellular biology of the yeast Saccharomyces cerevisiae: Genome dynamics, protein synthesis, and energetics (ed. J.R. Broach, J.R. Pringle, and E.W. Jones), pp. 407-521. Cold Spring Harbor Laboratory Press, Cold Spring Harbor, New York.

Rasmussen, S.W. and P.B. Holm. 1978. Human meiosis II. Chromosome pairing and recombination nodules in human sporocytes. Carlsberg Res. Commun. 43: 275-327.

Rockmill, B. and G.S. Roeder. 1990. Meiosis in asynaptic yeast. Genetics 126: 563-574.

- 1994. The yeast med1 mutant undergoes both meiotic homolog nondisjunction and precocious separation of sister chromatids. Genetics 136: 65-74.

Rockmill, B., J. Engebrecht, H. Scherthan, J Loidl, and G.S. Roeder. 1995. The yeast MER2 gene is required for meiotic recombination and chromosome synapsis. Genetics 141: 49-59.

Rose, M.D., P. Novick, J.H. Thomas, D. Botstein, and G.R. Fink. 1987. A Saccharomyces cerevisiae genomic plasmid bank based on a centromere-containing shuttle vector. Gene 60: $237-243$.

Rose, M.D., F. Winston, and P. Hieter. 1990. Methods in yeast genetics. Cold Spring Harbor Laboratory Press, Cold Spring Harbor, New York.

Rothstein, R. 1991. Targeting, disruption, replacement and allele rescue: Integrative DNA transformation in yeast. Methods Enzymol. 194: 281-301.

Scherthan, H., J. Loidl, T. Schuster, and D. Schweizer. 1992. Meiotic chromosome condensation and pairing in Saccharomyces cerevisiae studied by chromosome painting. Chromosoma 101: 590-595.

Schwacha, A. and N. Kleckner. 1994. Identification of joint molecules that form frequently between homologs but rarely between sister chromatids during yeast meiosis. Cell 76: $51-$ 63.

Shinohara, A., H. Ogawa, and T. Ogawa. 1992. Rad51 protein involved in repair and recombination in $S$. cerevisiae is a RecA-like protein. Cell 69: 457-470.

Sikorski, R. and P. Hieter. 1989. A system of shuttle vectors and yeast host strains designed for efficient manipulation of DNA in Saccharomyces cerevisiae. Genetics 122: 19-27.

Stack, S. and L. Anderson. 1986. Two-dimensional spreads of synaptonemal complexes from solanaceous plants. III. Recombination nodules and crossing over in Lycopersicon esculentum (tomato). Chromosoma 94: 253-258.

Story, R.M., D.K. Bishop, N. Kleckner, and T.A. Steitz. 1993. Structural relationship of bacterial RecA proteins to recombination proteins from bacteriophage $\mathrm{T} 4$ and yeast. Science 259: 1892-1896.

Sung, P. 1994. Catalysis of ATP-dependent homologous DNA pairing and strand exchange by yeast RAD51 protein. Science 265: 1241-1243.

Sym, M. and G.S. Roeder. 1994. Crossover interference is abolished in the absence of a synaptonemal complex protein. Cell 79: 283-292.

- 1995. Zipl-induced changes in synaptonemal complex structure and polycomplex assembly. J. Cell Biol. 128: 455466.

Sym, M., J. Engebrecht, and G.S. Roeder. 1993. ZIPl is a synaptonemal complex protein required for meiotic chromosome synapsis. Cell 72: 365-378.

Tishkoff, D.X., B. Rockmill, G.S. Roeder, and R.D. Kolodner. 1995. The sep1 mutant of Saccharomyces cerevisiae arrests in pachytene and is deficient in meiotic recombination. Genetics 139: 495-509.

von Wettstein, D., S.W. Rasmussen, and P.B. Holm. 1984. The synaptonemal complex in genetic segregation. Annu. Rev. Genet. 18: 331-413.

Weiner, B.M. and N. Kleckner. 1994. Chromosome pairing via multiple interstitial interactions before and during meiosis in yeast. Cell 77: 977-991.

West, S.C. 1992. Enzymes and molecular mechanisms of genetic recombination. Annu. Rev. Biochem. 61: 603-640. 


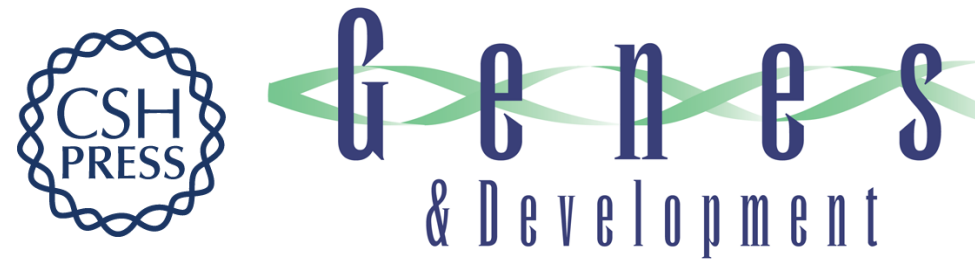

\section{Roles for two RecA homologs in promoting meiotic chromosome synapsis.}

B Rockmill, M Sym, H Scherthan, et al.

Genes Dev. 1995, 9:

Access the most recent version at doi:10.1101/gad.9.21.2684

References This article cites 58 articles, 28 of which can be accessed free at:

http://genesdev.cshlp.org/content/9/21/2684.full.html\#ref-list-1

License

Email Alerting

Service

Receive free email alerts when new articles cite this article - sign up in the box at the top right corner of the article or click here.

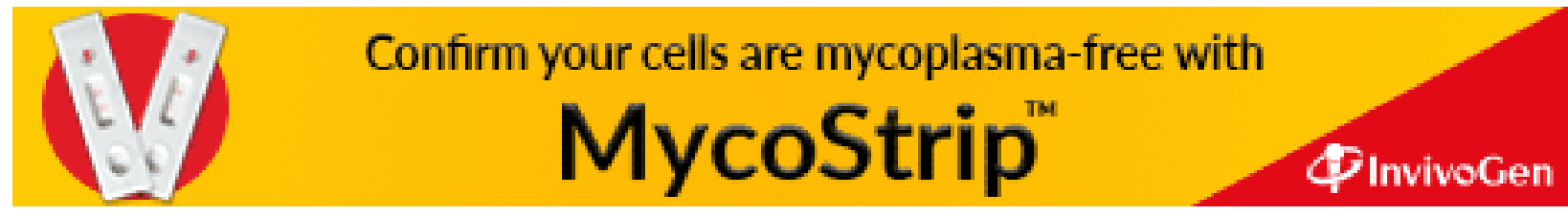

